\title{
RESÍDUOS DA CONSTRUÇÃO E DEMOLIÇÃO (RCD) APLICADOS EM CAMADAS DE BASE E SUB-BASE DE PAVIMENTOS
}

Maria de Jesus Melo Cerqueira ${ }^{1}$, UNINOVAFAPI Paulo Roberto Nunes de Farias ${ }^{2}$, UNINOVAFAPI Higor Jônathas Sena Dias Lopes ${ }^{3}$, UNINOVAFAPI João da Rocha Marinho Neto ${ }^{4}$, UNINOVAFAPI

\section{RESUMO}

O crescimento dos centros urbanos tem impulsionado o desenvolvimento do setor da construção civil, o qual tem gerado uma grande quantidade de resíduos sólidos, também conhecidos como Resíduos da Construção e Demolição (RCD), sendo possível a reutilização destes em camadas de pavimentos. Estes receberam atenção especial neste trabalho, onde buscou-se apresentar uma visão ampla sobre o atual estado da arte acerca da utilização de agregados reciclados de RCD em pavimentação, expondo seus principais aspectos, forma pela qual são gerados, impactos ambientais que provocam e principais aplicabilidades até então propostas, possibilitando, assim, que novas pesquisas sejam desenvolvidas. O objetivo buscado com esta pesquisa foi a reunião da bibliografia até então existente acerca da utilização de resíduos industriais na pavimentação, informando a comunidade sobre a necessidade da diminuição da geração de resíduos e a possibilidade de inserilos na pavimentação. Assim, neste trabalho, foi realizado um vasto estudo acerca da bibliografia existente relativa a aplicação desses resíduos em pavimentos, como meio de reunir os assuntos pertinentes a este tema e divulgar seus resultados.

Palavras-Chave: Pavimentação. Resíduos da Construção e Demolição. RCD. Cerâmicos.

\section{INTRODUÇÃO}

O século XX trouxe consigo um acentuado crescimento demográfico, mais aparente nos grandes centros urbanos, onde o desenvolvimento tecnológico aliado ao consumismo, induziram as pessoas a adquirirem mais bens de consumo. A junção destes fatores fez com que a quantidade de

\footnotetext{
${ }^{1}$ Graduanda em Engenharia Civil do Centro Universitário UNINOVAFAPI, mariadejesusmelocerqueira@gmail.com.

${ }^{2}$ Graduando em Engenharia Civil do Centro Universitário UNINOVAFAPI, pauloroberto.n.farias@gmail.com.

${ }^{3}$ Engenheiro Civil, Centro Universitário UNINOVAFAPI, higorjonathan@ gmail.com.

${ }^{4}$ Engenheiro Civil, CEUPI, joromaneto@gmail.com 
ANAIS CBCS 2019 | 3 a 5 de outubro de 2019 | Centro Universitário Santo Agostinho - Teresina - P

resíduos sólidos urbanos aumentasse de forma exponencial, os quais, na grande maioria dos casos, são dispostos sem nenhum tratamento específico, trazendo consequências negativas para o meio ambiente e para a saúde pública.

Com o aumento da geração de resíduos por parte das indústrias, alternativas corriqueiras como a deposição em aterros sanitários ou incineração passaram a não ser suficientes para destinar adequadamente todo esse montante de materiais. Neste contexto, técnicas como a reciclagem começaram a receber maior atenção, sendo impulsionadas por investimentos públicos e privados em pesquisas a cerca deste tema, tornando conhecidos os resíduos que possuem de fato uma aplicação proveitosa, e trabalhar, posteriormente, para a sua normatização.

Em vista a diminuir os impactos dos resíduos industriais, dentre os quais os resíduos da construção e demolição se destacam pela considerável parcela que representam do total de resíduos sólidos urbanos gerados, muitas aplicações vêm sendo propostas como meio de reinserilos nos processos produtivos da indústria.

Neste cenário, uma área que vem ganhando bastante atenção nesse contexto é a pavimentação, onde, na maior parte dos casos, os resíduos são trabalhados na forma de agregados reciclados e aplicados em suas camadas de base, sub-base e subleito.

Isto posto, foi realizado no presente trabalho, um levantamento bibliográfico do material intelectual até então existente à cerca da aplicação dos Resíduos da Construção e Demolição na pavimentação, tendo como foco principal os resíduos da construção e demolição (RCD) e os resíduos cerâmicos.

Ademais, fez-se o uso da revisão bibliográfica, usando-se de artigos, matérias e produções científicas disponíveis em livros, revistas, artigos, dissertações e teses.

\section{RESÍDUOS DA CONSTRUÇÃO E DEMOLIÇÃO}

Em vista a estabelecer procedimentos, diretrizes e critérios para uma eficaz minimização dos impactos ambientais negativos desencadeados pelo manejo inadequado dos RCD no Brasil, o 


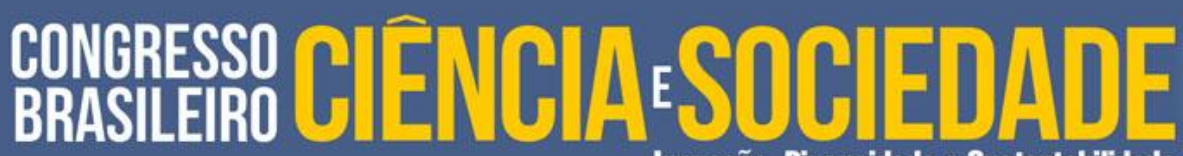

ANAIS CBCS 2019 | 3 a 5 de outubro de 2019 | Centro Universitário Santo Agostinho - Teresina - PI

CONAMA - Conselho Nacional do Meio Ambiente, no dia 5 de julho de 2002, criou a resolução n. 307, em vista a reduzir os impactos ambientais causados pela deposição inadequada do RCD.

A mesma resolução também propôs uma definição para tais resíduos (Art. $2^{\circ}$, Inciso IV), ao descrever que todo e qualquer resíduo proveniente de obras de construção, reformas, reparos e demolição, tendo como resultado de sua execução os tijolos, rochas, solos, metais, concreto em geral, tintas, madeiras e compensados, resina, gesso, vidros, telhas, plásticos, fiação elétrica, tubulações, entre outros (BRASIL, 2002).

\section{ASPECTOS GERAIS}

Segundo Freitas et al. (2016, apud ZANTA, 2008), nas grandes e médias cidades brasileiras a taxa de geração de resíduos da construção e demolição varia entre 400 a 700 kg/hab.ano, estimativa a qual depende de fatores como tamanho da cidade, momento econômico do país, época, entre outros. Os RCD equivalem, por si só, a mais da metade de todos os resíduos sólidos urbanos gerados, conforme mostrado no gráfico abaixo:

Gráfico 1 - Composição dos Resíduos Sólidos Urbanos.

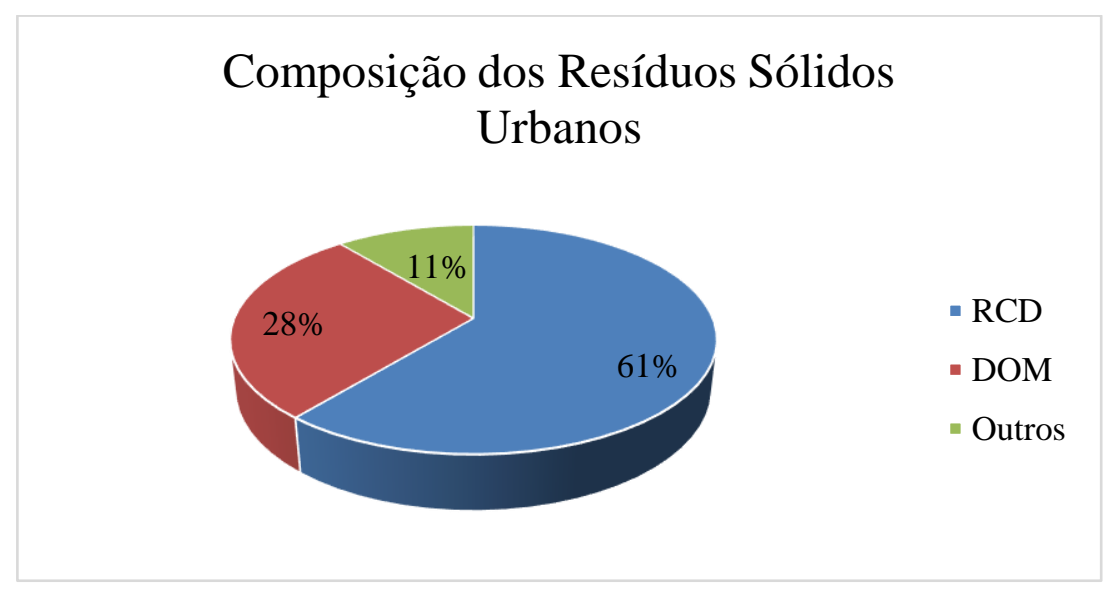

Fonte: (PINTO, 2004, p. 1).

O Gráfico 1 descreve bem a razão pela qual precisamos nos preocupar tanto com o manejo adequado dos RCD. De todos os resíduos gerados numa cidade de grande porte, apenas $28 \%$ são 
ANAIS CBCS 2019 | 3 a 5 de outubro de 2019 | Centro Universitário Santo Agostinho - Teresina - PI

provenientes de domicílios e $11 \%$ dos demais estágios das atividades humanas, sendo os $61 \%$ restantes originados a partir de um único setor, a indústria da construção civil.

Umas das desvantagens de utilizar os resíduos da construção e demolição é que não há áreas suficientes para receber o grande volume gerado, além de que o valor necessário para colocar em prática as usinas de reciclagem é muito alto, o que dificulta a implementação da gestão ambiental desses resíduos em muitas localidades (BRASILEIRO E MATOS, 2015).Com o grande aumento das pesquisas e utilização em casos reais da aplicação de resíduos da construção e demolição na pavimentação, a Associação Brasileira de Normas Técnicas (ABNT) sentiu a necessidade de normatizar tais procedimentos, sendo elaboradas as primeiras normas sobre o assunto na área em questão, sendo elas:

- NBR 15116 - Agregados reciclados de resíduos sólidos da construção civil - Utilização em pavimentação e preparo de concreto sem função estrutural - Requisitos;

- NBR 15115 - Agregados reciclados de resíduos sólidos da construção civil - Execução de camadas de pavimentação - Procedimentos.

\section{CLASSIFICAÇÃO DOS RESÍDUOS}

No Brasil, o órgão incumbido pela classificação dos Resíduos da Construção e Demolição é o Conselho Nacional do Meio Ambiente - Conama, o qual determina todos os critérios, diretrizes e procedimentos a serem seguidos para uma correta gestão desses resíduos.

Os resíduos da construção e demolição (RCD), de acordo com a resolução Conama n. 307 (BRASIL, 2002, p. 2) são divididos nas seguintes classes:

a) Classe A: é composta pelos seguintes resíduos reutilizáveis ou recicláveis como agregados:

- de construção, demolição, reformas e reparos de pavimentação e outras obras de infraestrutura, inclusive solos provenientes de terraplanagem;

- de construção, demolição, reformas e reparos de edificações: componentes cerâmicos (tijolos, blocos, telhas, placas de revestimento etc.), argamassa e concreto;

- de processo de fabricação e/ou demolição de peças pré-moldadas em concreto (blocos, tubos, meios-fios etc.) produzidos nos canteiros de obras;

b) Classe B: é composta por resíduos que tem a possibilidade de reciclagem, como plásticos, papeis, papelões, metais, vidros, madeiras e outros; 


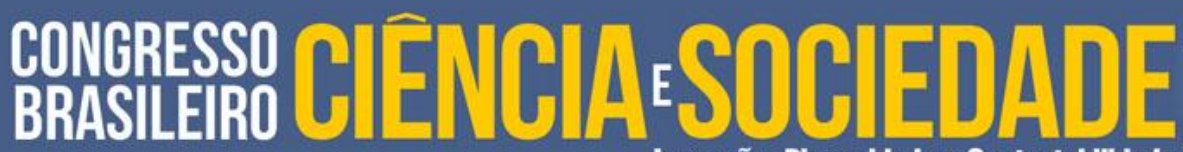 \\ $\rightarrow$ Inovação, Diversidlatle e Sustentahililitaile}

ANAIS CBCS 2019 | 3 a 5 de outubro de 2019 | Centro Universitário Santo Agostinho - Teresina - PI

c) Classe C: é composta por resíduos que ainda não possuem reciclagem ou recuperação por meio de tecnologias ou aplicações economicamente viáveis, como é o caso dos produtos procedentes do gesso;

d) Classe D: é composta pelos resíduos perigosos que são gerados nos processos construtivos de edificações, como tintas, solventes e óleos, ou, ainda, pelos resíduos gerados através de demolições e reformas de clínicas radiológicas e instalações industriais.

\section{IMPACTOS AMBIENTAIS}

A Resolução Conama n. 001 (BRASIL, 1986), Art $1^{\circ}$, define impacto ambiental como sendo:

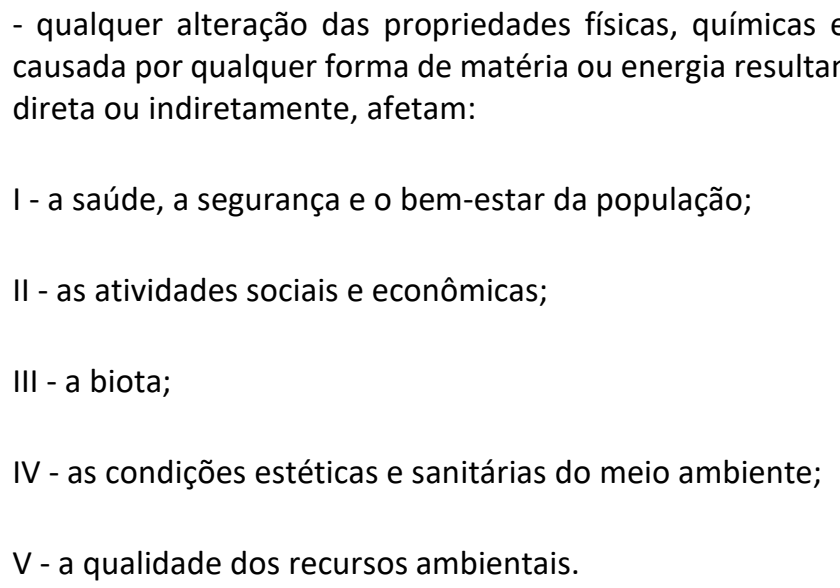

Isto posto, concluímos que os resíduos provenientes da construção e demolição de obras civis, causam, quando manejados de forma inadequada, um certo impacto ambiental, pois propiciam a proliferação de vetores de doenças; causam o entupimento dos sistemas de drenagem urbana; assoreiam lagos, rios e córregos; prejudicam a paisagem; comprometem o tráfego de pessoas e veículos, entre outros (CABRAL; MOREIRA, 2001).

Segundo Amadei et al.(2011), inúmeros são os impactos gerados pelos RCD nas mais diversas áreas:

- Ambiental: obstrução de rios que córregos que drenam as águas superficiais e ocupação de áreas naturais em baixadas, fundos de vale e terrenos desocupados;

- Econômica: custos elevados para a correta gestão dos RCD; 


\section{CONGQEESSOCIENCIAESOCIEDADE

ANAIS CBCS 2019 | 3 a 5 de outubro de 2019 | Centro Universitário Santo Agostinho - Teresina - PI

- Social: gestores e coletores de RCD desprovidos de conhecimentos técnicos suficientes para a preservação ambiental e sanitária;

- Sanitária: os resíduos da construção e demolição presentes em determinado ambiente propiciam o desenvolvimento de vetores que desempenham efeito deletério para o saneamento do local;

- Visual: comprometimento da paisagem local.

6. CASOS JÁ REGISTRADOS DE APLICAÇÕES DE RCD EM PAVIMENTAÇÃO NO BRASIL E NO MUNDO

Em 1984 a primeira via do Estado de São Paulo contendo agregados reciclados de RCD em sua composição, foi pavimentada, a partir de uma parceria entre o Estado e o Instituto de Pesquisas Tecnológicas (IPT), para o acompanhamento da obra. Um pouco mais tarde, em Belo Horizonte, no ano de 1996, algumas avenidas já vinham sendo pavimentas com esse tipo de resíduo em sua composição, sendo empregados em todas as camadas do pavimento (MOTTA, 2005, apud BAGANTINI, 2011).

Já em Goiânia, pavimentaram uma pista experimental com agregado reciclado de $R C D$, onde analisaram seu uso nas diferentes camadas do pavimento - reforço de subleito, sub-base e base (MENDES et al., 2004, apud QUEIROZ E MELO, 2011).

Em países europeus, asiáticos e norte-americanos, a utilização de resíduos provenientes de obras civis é ainda maior. No Reino Unido, por exemplo, uma das mais importantes aplicações é o emprego da brita calcária beneficiada, utilizada nas camadas de sub-base dos pavimentos. (LEITE, 2007).

Nos Estados Unidos, uma pista de testes foi construída na Pista Circular da University of Central Florida, a qual continha nove seções-tipo distintas, sendo cinco delas de pavimento rígido e quatro de pavimento flexível, todas utilizando agregado reciclado de concreto (LEITE, 2007).

\section{APLICAÇÕES DOS RESÍDUOS DA CONSTRUÇÃO E DEMOLIÇÃO NA PAVIMENTAÇÃO}


ANAIS CBCS 2019 | 3 a 5 de outubro de 2019 | Centro Universitário Santo Agostinho - Teresina - PI

Diversas pesquisas já propostas até então com a temática da reutilização dos resíduos da construção e demolição como agregados reciclados mostram um amplo leque onde esses resíduos podem ser reinseridos, sendo um dos componentes mais notório desse leque a pavimentação.

Segundo a ABRECON (2015), umas das formas mais simples para reciclagem dos resíduos da construção e demolição é a sua implementação nas camadas de base, sub-base ou revestimento primário de pavimentos, em misturas do agregado reciclado com o solo ou ainda na forma de brita corrida. Para exercer tal função, o RCD precisa ser processado em mecanismos de britagem/trituração para que alcancem a granulometria de projeto.

A implementação do uso de agregados reciclados de RCD, como substituto do agregado natural em concreto asfáltico, por exemplo, já é uma realidade em muitos lugares. Umas das grandes vantagens dessa substituição, é que, por exemplo, a utilização de seixo em misturas asfálticas a quente o garantem alta resistência mecânica, porém, como boa parte do seixo existente provém de rios e lagos, substituí-los nas misturas asfálticas por agregados reciclados já diminuiria significativamente impactos como desmatamento em áreas de extração do material e morte da vida aquática (BRASILEIRO E MATOS, 2015).

Em seus estudos, Bagantini (2011) avaliou o comportamento de pavimentos de vias urbanas onde as camadas de materiais naturais, como a brita graduada, foram substituídos por agregados reciclados de $R C D$, devidamente classificado. Para seus testes, utilizou uma pista experimental de testes de cargas fazendo uso de resíduos da construção e demolição, os quais formavam camadas de diferentes espessuras. A primeira camada continha $45 \mathrm{~cm}$ de resíduos de diâmetro máximo $5 \mathrm{~cm}$, seguida por $20 \mathrm{~cm}$ de brita graduada e $70 \mathrm{~cm}$ de blocos de concreto. A segunda camada continha $55 \mathrm{~cm}$ de RCD, seguida por $10 \mathrm{~cm}$ de brita graduada e $70 \mathrm{~cm}$ de blocos. A terceira e última camada, não continha brita graduada, mas $\operatorname{sim} 65 \mathrm{~cm}$ de RCD e $70 \mathrm{~cm}$ de blocos de concreto.

Após realizar uma análise mecanística das estruturas descritas, Bagantini (2011) pôde observar que, entre as três camadas, a primeira apresentou melhores resultados ao suportar um volume de tráfego $\mathrm{N}$ da ordem de $7 \times 10^{4}$ no tocante à análise da fadiga por meio das deformações admissíveis nas três camadas da pista. Na análise do rompimento por cisalhamento da camada de 
ANAIS CBCS 2019 | 3 a 5 de outubro de 2019 | Centro Universitário Santo Agostinho - Teresina - PI

subleito, mais uma vez a primeira camada se sobressaiu, mostrando-se superior as demais ao suportar um volume de tráfego $\mathrm{N}$ da ordem de $6 \times 10^{4}$. Com relação ao módulo de resiliência, ele observou, também, que a capacidade estrutural de uma camada de 28 centímetros de resíduos de diâmetro máximo de 2 polegadas equivale a uma camada de $20 \mathrm{~cm}$ de brita graduada, tomando como base um volume de tráfego não maior que $\mathrm{N}=10^{5}$.

Queiroz e Melo (2011) estudaram a viabilidade técnica de agregados de resíduo misto (ARM) para uso em pavimentação. Em seus testes, o índice de suporte Califórnia (ISC) mínimo obtido foi de 19\%, permitindo, segundo a NBR 15116, o uso dos agregados reciclados em reforço de subleito.

Em São Paulo, Motta (2005) analisa laboratorialmente aspectos físicos do agregado reciclado de RCD aplicado em pavimentação de baixo volume de tráfego. Foi verificado que os agregados reciclados absorvem muito mais água (aproximadamente $8 \%$ ) do que os agregados usuais (cerca de 2\%), uma vez que sua porosidade é elevada em relação aos mesmos, implicando numa maior necessidade de água por ocasião da compactação. Além disso, verificou-se também que a forma do agregado reciclado de RCD é cúbica, o que favorece ainda mais a sua aplicação em pavimentação.

Motta (2005) conclui, ainda, que os valores para o índice de suporte Califórnia (ISC) do agregado reciclado encontra-se acima de $75 \%$ (de forma aproximada) para os diferentes tempos de cura observados, satisfazendo, assim, os limites da NBR 15115, a qual diz que o ISC de agregados reciclados aplicados em camadas de reforço de subleito, sub-base e base tem de ser superior a $60 \%$ (isto para baixo volume de tráfego), 20\% e 12\%, respectivamente.

Amadei (2011) também analisou o comportamento dos resíduos da construção e demolição na pavimentação, porém, em pavimentos intertravados. Em seus estudos, testou substituições parciais do agregado miúdo natural pelo agregado reciclado de RCD, nos teores de $25 \%, 30 \%, 35 \%$, $40 \%, 45 \%$ e $50 \%$. Concluiu-se, com os testes, que o teor de $25 \%$ de substituição apresentou resultados dentro dos limites estabelecidos pela NBR 9790 (1987), atingindo valores superiores a 35 Mpa ao completar 28 dias. Os teores de 30\% e 35\% não atingiram a resistência estabelecida pela norma, porém, apresentarem resultados aceitáveis para utilização em locais com baixa solicitação de tráfego. 
ANAIS CBCS 2019 | 3 a 5 de outubro de 2019 | Centro Universitário Santo Agostinho - Teresina - P

\section{METODOLOGIA}

O presente trabalho trata-se de uma revisão bibliográfica baseada em conteúdos já existentes, composta basicamente de artigos científicos, revistas, livros e algumas matérias encontradas em sítios on-line. Desta forma, aborda-se a análise da aplicação de resíduos industriais (resíduos da construção e demolição e resíduos cerâmicos) na pavimentação, tomando como base artigos publicados sobre o assunto nas línguas portuguesa e inglesa.

\section{CONSIDERAÇÕES FINAIS}

O crescimento dos grandes centros urbanos tem potencializado a geração de resíduos, principalmente os oriundos da indústria da construção civil. Estes, além de representarem uma grande parcela do total de Resíduos Sólidos Urbanos gerados, possuem o agravante de serem inertes. Para tanto, medidas deverão ser tomadas de modo a minimizar seus impactos negativos, indo desde a reutilização desses resíduos em novas atividades até melhorias na gestão de obras.

Outrossim, o emprego de agregados de resíduos da construção e demolição reciclados em camadas de base de pavimentos já não é uma novidade, sendo uma prática já difundida em algumas partes do Brasil e do mundo. Porém, como a pavimentação é um mercado quase que totalmente comandado pelo setor público, para que consideráveis quantidades de RCD sejam reciclados faz-se necessário uma diversificação do mercado, de modo a atingir um maior número de clientes.

Para a maior popularização da utilização de RCD e resíduos cerâmicos na pavimentação, fazse necessário, entre outros fatores, o incentivo à pesquisa, para que assim sejam obtidos dados e especificações cada vez mais apurados sobre as propriedades desses coprodutos, ainda muito julgados pelos céticos, representando, assim, uma barreira a sua popularização.

Na maior parte das pesquisas aqui referenciadas, tanto os Resíduos da Construção e Demolição quanto os Resíduos Cerâmicos atingiram valores considerados aceitáveis para sua utilização na pavimentação, seja pavimento rígido, flexível, intertravado, entre outros, constituindo uma ótima maneira de reduzir a quantidade de resíduos depositados em áreas indevidas, e diminuir a quantidade de matérias-primas extraídas para a fabricação destes insumos. 
ANAIS CBCS 2019 | 3 a 5 de outubro de 2019 | Centro Universitário Santo Agostinho - Teresina - P

Por fim, fica como sugestão para futuras pesquisas a realização de revisões bibliográficas sobre a reutilização de outros resíduos industriais também aplicados em pavimentação, como as escórias de autoforno e as cinzas volantes, expondo suas principais características, a forma pela qual são gerados, os impactos que provocam e suas principais aplicações até então conhecidas.

\section{REFERÊNCIAS}

AMADEI, Daysa; PEREIRA, Juliana; SOUZA, Rafael; MENEGUETTI, Karin. A Questão dos Resíduos de Construção Civil: Um Breve Estudo da Arte. Revista NUPEM, Campo Mourão, v.3, n.5. Brasil, 2011. AMADEI, Deysa Ione Braga. Avaliação de Blocos de Concreto Para Pavimentação Produzidos com Resíduos de Construção e Demolição do Município de Juranda/PR. Dissertação (Mestrado) Universidade Estadual de Maringá, Departamento de Engenharia Civil, Programa de Pós-graduação em Engenharia Urbana, Maringá, 2011.

ABRELPE. Panorama dos Resíduos Sólidos no Brasil. 2013. Disponível em: <http://www. abrelpe.org.br/ Panorama/panorama2012.pdf>. Acesso em: 17 de julho de 2018.

ASSOCIAÇÃO BRASILEIRA DE NORMAS TÉCNICAS. NBR 10.004: resíduos sólidos - classificação. Rio de Janeiro, 2004.

BAGATINI, Felipe. Resíduos da construção civil: aproveitamento como base e sub-base na pavimentação de vias urbanas. 2011. 70 p. Trabalho de Conclusão de Curso (Engenharia Civil) Universidade Federal do Rio Grande do Sul, Porto Alegre, Rio Grande do Sul.

BRASIL. Ministério do Meio Ambiente. Conselho Nacional do Meio Ambiente. Resolução n. 307, de 5 de julho de 2002. Estabelece diretrizes, critérios e procedimentos para a gestão dos resíduos da construção civil. Brasília, $2002 . \quad$ DF, Disponível em: <http://www.mma.gov.br/port/conama/legiabre.cfm?codlegi=307>. Acesso em: 01 de julho de 2018.

BRASIL. Ministério do Meio Ambiente. Conselho Nacional do Meio Ambiente. Resolução n. 001, de 23 de janeiro de 1986. Estabelece definições, as responsabilidades, os critérios básicos e as diretrizes gerais para uso e implementação da Avaliação de Impacto Ambiental como um dos instrumentos 
ANAIS CBCS 2019 | 3 a 5 de outubro de 2019 | Centro Universitário Santo Agostinho - Teresina - PI

da Política Nacional do Meio Ambiente. Brasília, DF, 1986. Disponível em: < http://www.mma.gov.br/port/conama/res/res86/res0186.html >. Acesso em: 15 de julho de 2018. BRASILEIRO, L. L; MATOS, J. M. E. Revisão bibliográfica: reutilização de resíduos da construção e demolição na indústria da construção civil. Revista Cerâmica, São Paulo, v. 61, n. 358. Brasil, 2015. CABRAL, A. E. B; MOREIRA, K. M. V. Manual sobre Resíduos Sólidos da Construção Civil. Sinduscon CE, Fortaleza. Brasil, 2001.

CAVALCANTI, J. E. A década de 90 é dos resíduos sólidos. 2004. Disponível em: <http://riosvivos.org.br/a/Noticia/A+decada+de+90+e+dos+residuos+solidos/3223>. Acesso em: 11 de julho de 2018.

FREITA, C. L. V; SANTOS, V. M. L; JÚNIOR, J. E. S; SILVA, T. C. C. Reciclagem de resíduos da construção e demolição (RCD): um estudo de caso na usina de beneficiamento de resíduos de Petrolina - PE. Revista RGSA, São Paulo, v. 10, n. 1. Brasil, 2016.

LEITE, F. C. Comportamento mecânico de agregado reciclado de resíduo sólido da construção civil para emprego em camadas de base e sub-base de pavimentos. 2007. 198 p. Dissertação (Mestrado em Engenharia de Transportes) - Departamento de Engenharia de Transportes, Escola Politécnica, Universidade de São Paulo, São Paulo.

LERIPIO, A. A. Gerenciamento de resíduos.2004. Disponível em: <http://www.eps.ufsc.br/ |gqa/Coferecidos.html>. Acesso em: 11 de julho de 2018.

MACEDO, R.J.F.; Santos, R.S.; Araújo, M.S.G.; Oliveira, J.F.; Marinho, R.M.M. Caracterização dos resíduos das indústrias cerâmicas estruturais da Região do Cariri. Caderno de Cultura e Ciência, Ano VII, v.11, n.2, dezembro, 2012.

MENDES, et al.. Parâmetros de uma pista experimental executada com entulho reciclado. In: ReuniãoAnual de Pavimentação, 35a. RAPv. 2004. Anais, Cd-rom. Rio de Janeiro.

MOTTA, R. S. Estudo laboratorial de agregado reciclado de resíduo sólido da construção civil para aplicação em pavimentos de baixo volume de tráfego. 2005. 134 p. Dissertação (Mestrado em Engenharia de Transportes) - Universidade de São Paulo, São Paulo. 


\section{CONGQEESSOCIENCIAESOCIEDADE

ANAIS CBCS 2019 | 3 a 5 de outubro de 2019 | Centro Universitário Santo Agostinho - Teresina - PI

PINTO, Tarcísio de Paula. Metodologia para a gestão diferenciada de resíduos sólidos da construção urbana. 2004.Tese (Doutorado) - Escola Politécnica da Universidade de São Paulo, São Paulo, 1999.

QUEIROZ, B. O; MELO, R. A. Agregados reciclados de resíduos sólidos da construção civil: uma alternativa de materiais para a pavimentação. In: VIII Encontro Tecnológico da Engenharia Civil e Arquitetura. 2011, Maringá. Anais: Maringá, 2011.

SILVA, Mônica Maria Pereira da. Avaliação de perdas de blocos cerâmicos em Pernambuco: Da indústria ao canteiro de obras. 2007. Dissertação (Mestrado) - Curso de Mestrado em Engenharia Civil, Departamento de Pró-reitoria Acadêmica, Universidade Católica de Pernambuco, Recife, 2007. SOUZA, Ubiraci Lemes. Perdas de materiais nos canteiros de obras: a quebra do mito. Revista Qualidade na Construção, v. 2, n. 13. São Paulo, 1998.

TERA. O que é resíduo industrial e quais são suas classificações?.2016. Disponível em: <https://www.teraambiental.com.br/blog-da-tera-ambiental/o-que-e-residuo-industrial-e-quaissao-suas-classificacoes>. Acesso em: 11 de julho de 2018.

VIEIRA, Geilma; DAL MOLIN, Denise. Resistência e durabilidade de concretos produzidos com agregados reciclados provenientes de Resíduos de Construção e Demolição. Revista Engenharia Civil, Uminho, v. 19. Uminho, Portugal, 2004.

VGRESÍDUOS. Resíduos industriais: porque é importante fazer o descarte correto?.2018. Disponível em: <https://www.vgresiduos.com.br/blog/descarte-correto-residuos-industriais/>. Acesso em: 11 de julho de 2018.

ZANTA, V. M. (2008) Coordenação Geral Núcleo Regional do Nordeste- (Nurene). Resíduos sólidos: gerenciamento e reciclagem de resíduo de construção e demolição RCDs. [Guia Do Profissional em Treinamento Níveis 1 E 2]. Salvador: ReCESA. 